\title{
Bringing 3D visualization into the online research article
}

\author{
IJsbrand Jan Aalbersberg ${ }^{\mathrm{a}}$, Pilar Cos Alvarez ${ }^{\mathrm{a}}$, Julien Jomier ${ }^{\mathrm{b}}$, Charles Marion ${ }^{\mathrm{b}}$ and \\ Elena Zudilova-Seinstra ${ }^{\mathrm{a}, *}$ \\ ${ }^{\mathrm{a}}$ Elsevier, Amsterdam, The Netherlands \\ E-mails: \{IJ.J.Aalbersberg, P.Cosalvarez,E.Zudilova-Seinstra\}@elsevier.com \\ ${ }^{\mathrm{b}}$ Kitware SAS, Lyon, France \\ E-mails: \{julien.jomier, charles.marion\}@kitware.com
}

\begin{abstract}
The ICT revolution of the last decade impacts scientific publishing and changes the format in which articles are delivered and how results of scientific research are communicated. With researchers increasingly generating and referencing 3D data in their articles, the ability to accurately visualize that data in online publications has become extremely important. To address growing demands in online 3D visualization across various scientific domains and journals, Elsevier and Kitware launched a project to develop a 3D visualization infrastructure supporting different data formats, visualization techniques, and interaction styles for online scientific publishing. In this paper, we describe the expandable Hybrid Visualization Platform and five domain-specific 3D visualization viewers and present results of an author feedback survey. The presence of interactive 3D viewers embeddable in online articles is a key example of how the digital article format adds value to scientific communication and how it helps readers to better understand research findings and re-use the associated 3D data.
\end{abstract}

\section{Introduction}

With the transition from print to online publishing, the layout and presentation of scientific articles have remained relatively unchanged, still following a centuries-old format. Meanwhile, the number of available scientific supplementary content on the Internet grows exponentially every year [7]. Integration of all this scientific information with the article is necessary to keep publishing efficient and to maintain control over the process [24].

Authors in many different scientific disciplines deal with 3D data. 3D visualization is an important tool for understanding complex structures, dynamic simulations, and research discoveries [28]. In the traditional print-based scientific publication, 3D models are "flattened" into static 2D images, which significantly reduce the value of the author's analysis and the level of understanding by the reader, as each such 2D image can only capture one specific projection of a 3D object. Hence, embedding 3D visualization tools in online research articles is becoming extremely important [4].

To support this need, Elsevier [14] offers several online 3D viewers inside online scientific articles in various journals on ScienceDirect. When embedded in the article, 3D visualization tools allow readers to interactively explore 3D objects without interrupting the reading process. The functionality of each $3 \mathrm{D}$ viewer is carefully thought out in order to support domain-specific needs that are required for getting

\footnotetext{
*Corresponding author. E-mail: E.Zudilova-Seinstra@elsevier.com.
} 
sufficient understanding of the 3D data within each specific research community. When a thorough investigation is needed, the reader can download the original dataset provided by the author and explore it using visualization tools that he/she normally utilizes in his/her every-day activities.

In this paper, we will describe some of our technological advances in bringing 3D visualizations into research articles published in Elsevier journals. We will explain how molecular structures, neuroimaging data, archaeological models, etc. are visualized inside the article to create a faster understanding of and a deeper insight into the research described.

\section{The Hybrid Visualization Platform for online 3D visualization}

To address growing demands in online 3D visualization across various journals and scientific domains, Elsevier launched a project to build a 3D visualization infrastructure supporting different data formats, as well as domain-specific visualization techniques and interaction styles. The goal of this project is to provide a generic online visualization environment that allows ScienceDirect users to view and interact with the 3D datasets that are submitted with an Elsevier article - irrespective of the user access platform, including mobile phones, tablets, laptops and desktops. This project is built on a close collaboration between Elsevier and Kitware SAS [18], which serves as the 3D visualization service provider.

The resulting Hybrid Visualization Platform (Fig. 1) is now available and provides a flexible visualization solution that combines local (WebGL) and remote (ParaViewWeb) rendering techniques, allowing the user to view and interact with small to massive 3D datasets on a large number of devices, without any additional plug-in. Depending on the web browser and the size of the 3D data, the most optimal rendering support is offered such that a smooth and real-time user interaction with even very large 3D models is guaranteed.

The hybrid visualization system automatically selects the optimal visualization technology based on several factors: the rendering capabilities of the device, the size of the dataset, and the potential latency of the communication bandwidth [17]. The selection is done automatically by the server during the initial request:

- Local rendering uses the WebGL technology [8] and therefore transmits the full datasets to the client to provide the rendering. While the rendering is smoother and more interactive, only for small datasets the data transmission can be achieved with reasonable speed. Moreover, WebGL is not currently supported by all the devices and just started to be supported by the Internet Explorer web browser.

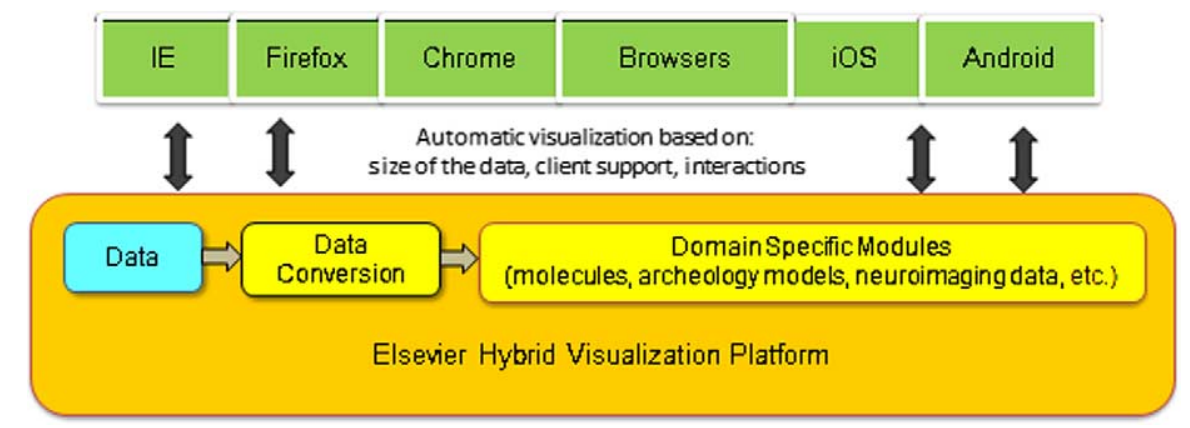

Fig. 1. The architecture of the expandable Hybrid Visualization Platform. (Colors are visible in the online version of the article; http://dx.doi.org/10.3233/ISU-140721.) 


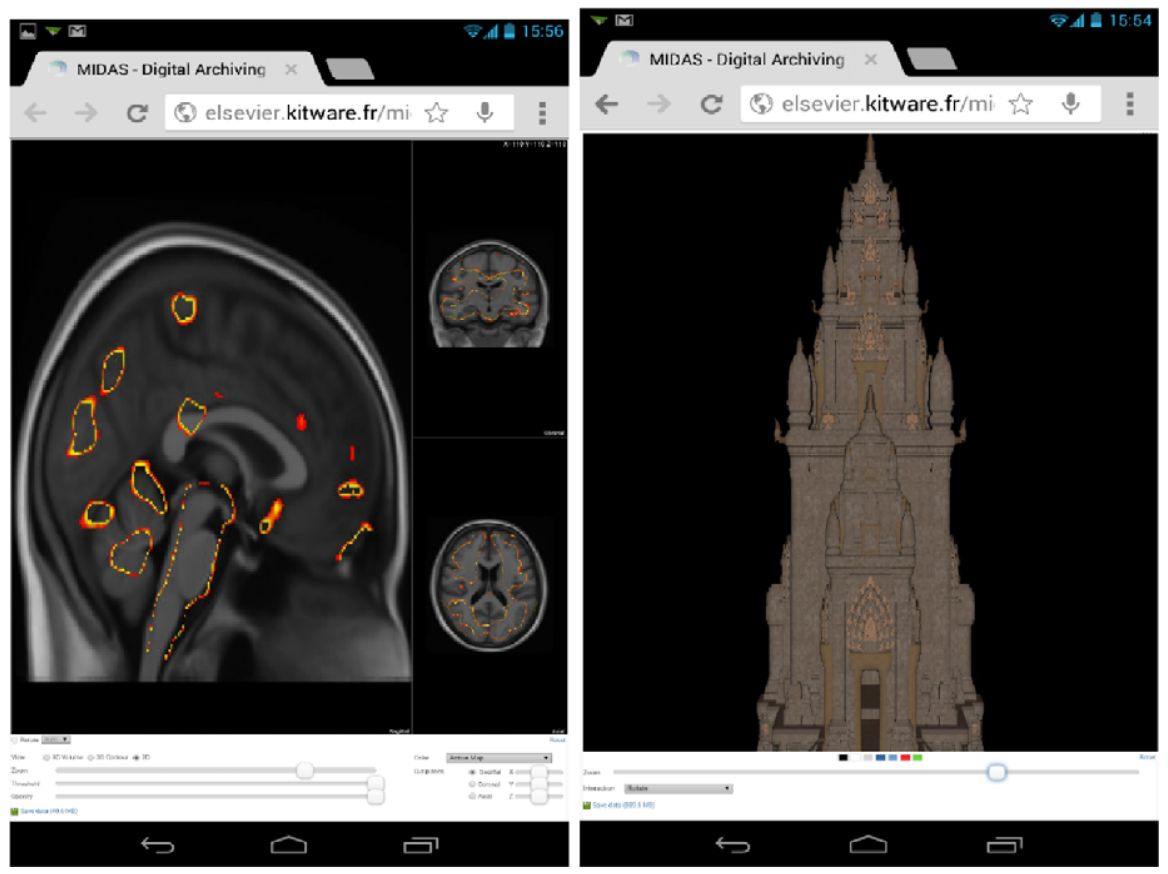

Fig. 2. Representations of how the 3D viewers appear on mobile devices: 3D neuroimaging viewer (left) and 3D archaeological viewer (right). (Colors are visible in the online version of the article; http://dx.doi.org/10.3233/ISU-140721.)

- Remote rendering is performed on a dedicated server, where only the rendered images are sent to the reader's device. This allows large datasets to be rendered on minimal devices, such as smartphones (Fig. 2) and tablets. In order to reach optimal interactivity and low latency, special communication protocols based on WebSockets have been implemented.

In both cases, one should notice that the interactivity of the 3D manipulation is directly correlated to the latency and speed of the connection between the device and the server.

The current platform is based on several open-source toolkits, such as The Visualization Toolkit [23], ParaView [6], and the data sharing platform MIDAS [16]. The deployed system is currently available on a wide range of web browsers including smartphones and tablets. Currently the platform has been tested on iOS and Android devices.

\section{A variety of interactive $3 D$ viewers}

The benefits of 3D visualization are many and can be applied to a wide variety of fields. On ScienceDirect, the following five 3D viewers are available on the article page:

- 3D molecular viewer;

- 3D archaeological viewer;

- 3D neuroimaging viewer;

- Generic U3D viewer;

- 3D Virtual Environments. 
Molecular visualizations help to understand mechanisms at the microscopic scale that are responsible for macroscopic behavior of materials. 3D archeological visualizations serve as a basis for preservations and interactive presentations of the cultural heritage. 3D neuroimaging allows the readers to better understand the brain functioning in health and diseases. Architectural 3D visualizations give the reader the ability to understand the most complex projects without having to analyze complex architectural drawings and schema.

All 3D viewers are displayed next to the relevant online articles, in the right-hand side panel; it is also possible to open viewers in full-screen mode. The 3D models and data are uploaded as supplementary files to the Elsevier Editorial System by the authors, after which each dataset is pre-processed once the article is accepted.

\subsection{D molecular viewer}

The 3D molecular viewer, as shown in Fig. 3, visualizes protein structures, ribosomes, chemical compounds, crystallographic data, etc. It supports PDB, PSE and MOL/MOL2 data formats, and it allows the 3D molecular models to be explored using the two most common visualization techniques: "ribbons" and "balls-and-sticks" [2]. Using the viewer, the reader can: browse through 3D molecular models using the thumbnail menu on top, zoom into each model, rotate and pan the model, change display settings, and download original data files. It is also possible to view molecular models in the 3D stereo mode using anaglyph glasses. The 3D molecular viewer is currently available in 20 Elsevier journals from various fields of Life Sciences (see, e.g. [10]).
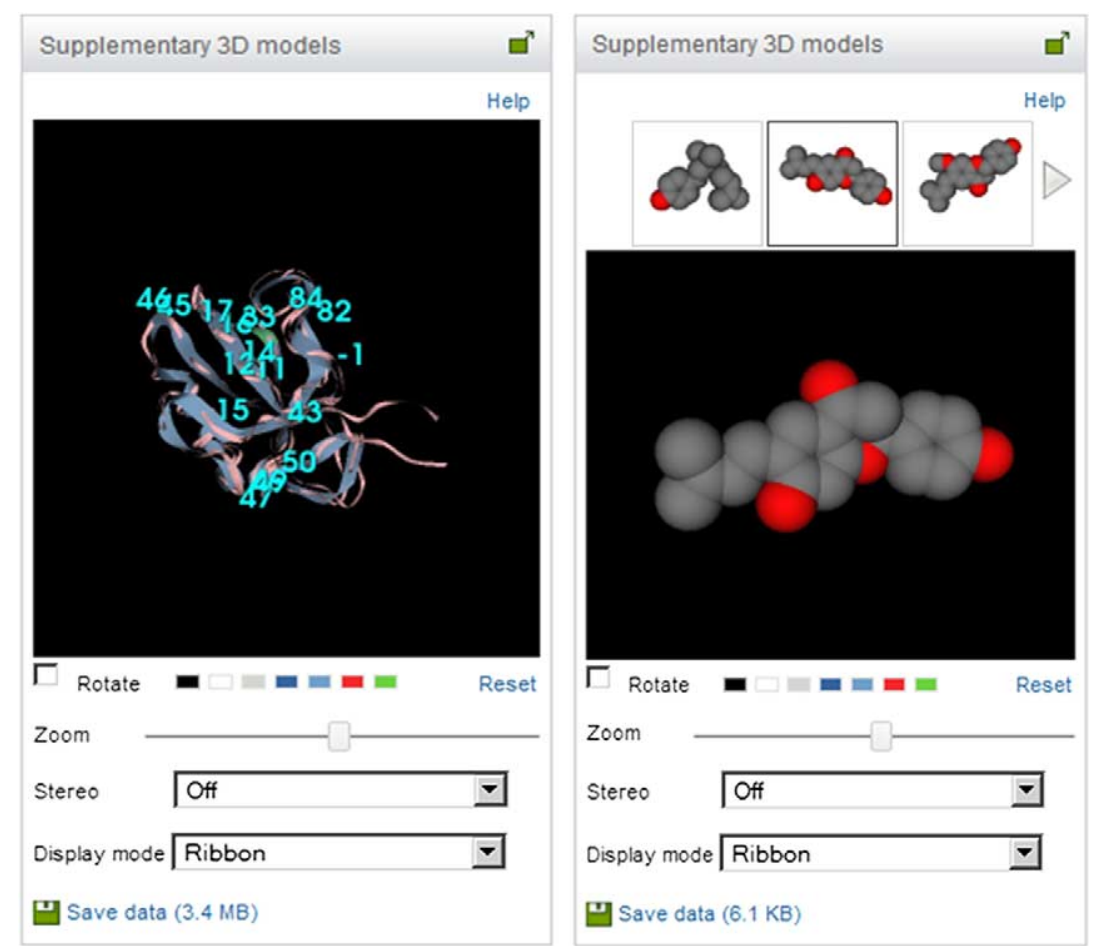

Fig. 3. Two examples of 3D molecular structures. (Colors are visible in the online version of the article; http://dx.doi.org/ 10.3233/ISU-140721.) 
In order to allow fast rendering of very large molecules a specific rendering mapper has been implemented. This mapper combines two rendering techniques: impostors and geometry instancing. Impostors are usually implemented to lower the number of triangles rendered in a scene by rendering the image of an object instead of rendering the object geometry [20]. To trick the user, the semi-transparent image of the object (the billboard) is drawn on a quad and then dynamically oriented to face the camera. Geometry instancing is a GPU capability which consists of rendering multiple copies of a given mesh in a single API call.

Our implementation instantiates the simple geometry of a billboard and for each instance, the position and the orientation of the instance is computed on the GPU. Thanks to these two techniques, our mapper is able to render millions of atoms interactively. A specific mapper has also been developed to allow for ribbon rendering.

\section{2. $3 D$ archaeological viewer}

The 3D archaeological viewer visualizes models submitted in PLY and OBJ formats [1]. To properly display 3D data, with effective support for texture and material properties, the surface rendering technique is applied. Using the viewer, the reader can scale the 3D model described in the article, rotate and pan the model using a mouse, use the auto-rotate option, change display settings, and download original data files. Two examples of 3D archaeological models visualized with the viewer are shown in Fig. 4.

This 3D archaeological viewer has been developed to support the new Elsevier Journal "Digital Applications in Archaeology and Cultural Heritage" (DAACH). DAACH offers scientists the opportunity of

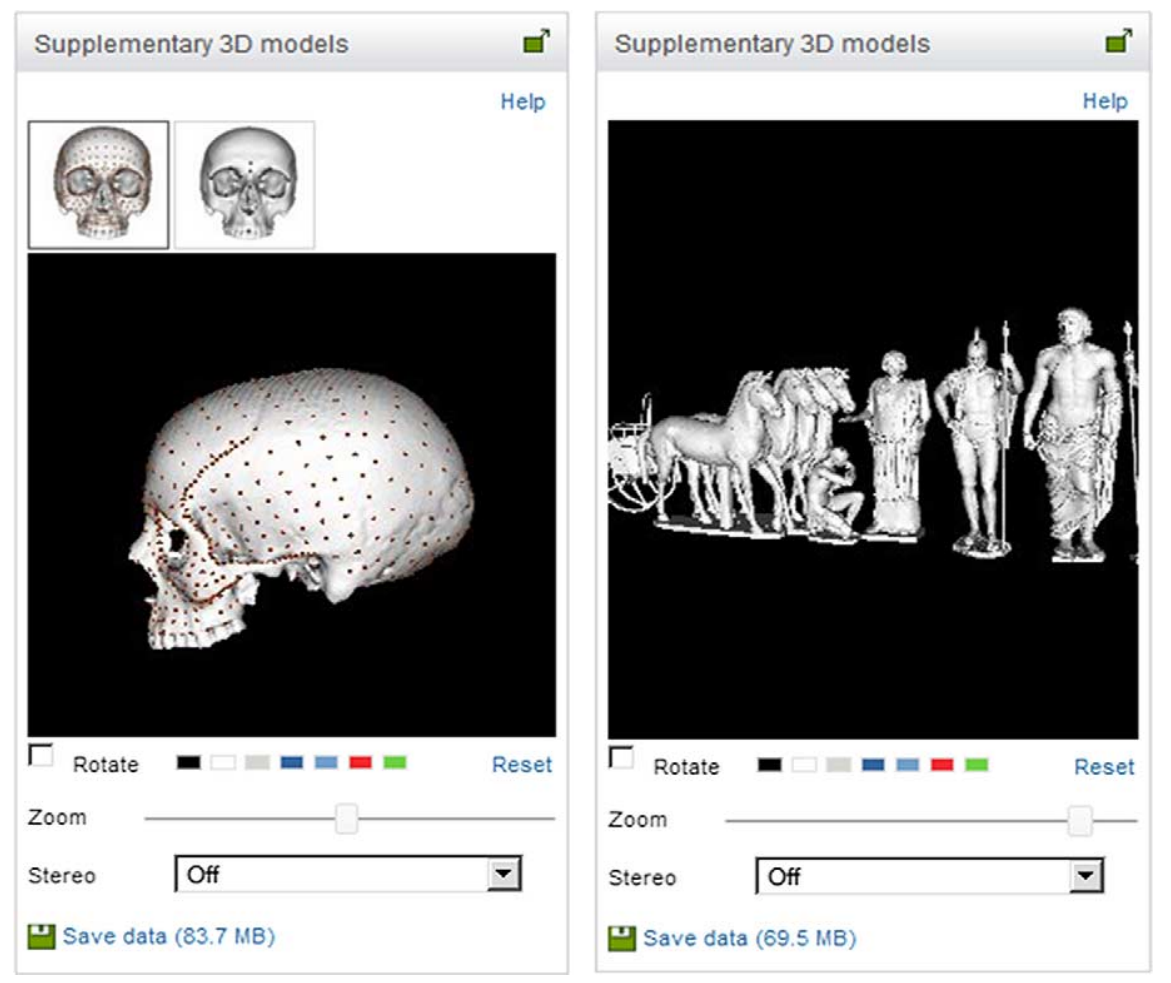

Fig. 4. Two examples of 3D archaeological models. (Colors are visible in the online version of the article; http://dx.doi.org/ 10.3233/ISU-140721.) 
publishing their models online with full interactivity, so that readers can explore them at will. The journal is unique in that its focus is on the application of 3D modeling to cultural heritage (see, e.g. [27]).

\section{3. $3 D$ neuroimaging viewer}

The 3D neuroimaging viewer visualizes analyzed neuroimaging data in NIfTI format [12]. This viewer enables the reader to browse through available neuroimaging datasets, zoom, rotate and pan the 3D brain reconstruction, cut through the volume, change opacity and color mapping, switch between 3D and 2D projected views, and download the data [3]. The viewer has been developed in close collaboration with neuroscientists and supports the most important capabilities as selected through an online survey with 223 neuroscientists.

The 3D neuroimaging viewer also integrates a specific visualization mapper which automatically combines a master volume of the brain with functional magnetic resonance images (fMRI). This mapper allows the readers to visualize and interact in real-time with the 3D brain volume and highlight the regions of interest in the brain (Fig. 5). The rendering is done using a volume rendering technique, which allows to adjust automatically the visualization of the different structures: white matter, gray matter, and cerebrospinal fluid.

The 3D neuroimaging viewer is currently available in 35 Elsevier journals in neuroscience, psychiatry, neurology and psychology (see, e.g. [11]).

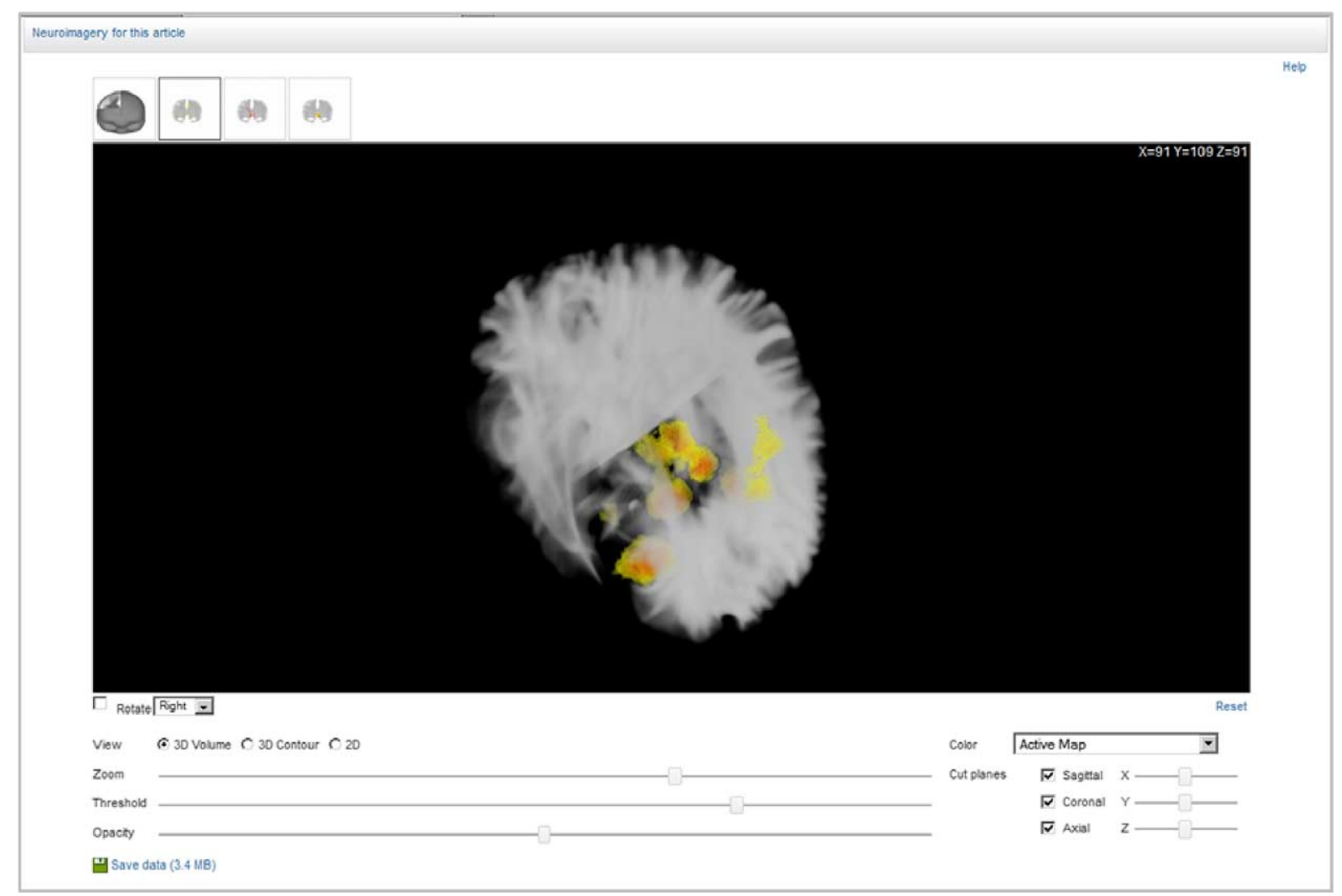

Fig. 5. The 3D neuroimaging viewer in the full-screen mode. (Colors are visible in the online version of the article; http://dx.doi.org/10.3233/ISU-140721.) 

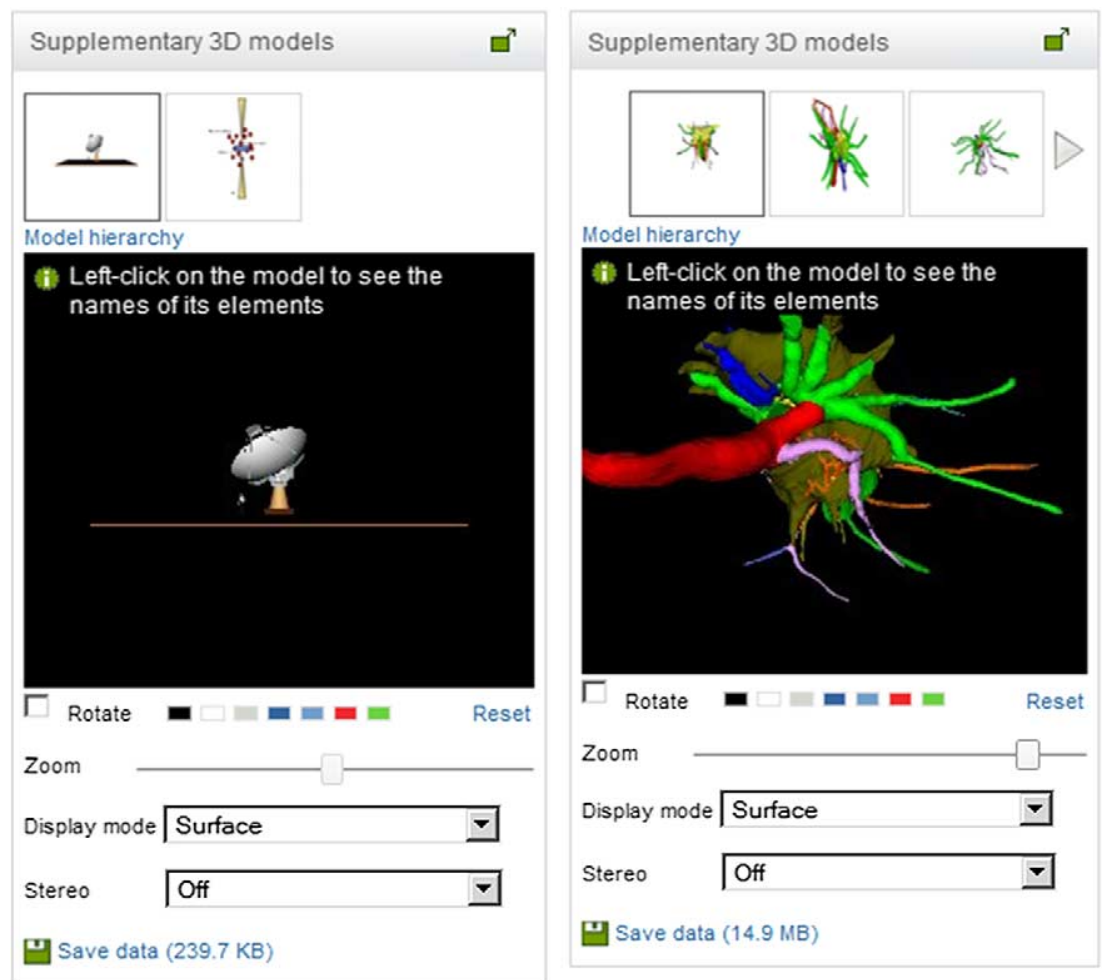

Fig. 6. Two examples of U3D models from: astrophysics (left) and neuroscience (right). (Colors are visible in the online version of the article; http://dx.doi.org/10.3233/ISU-140721.)

\subsection{Generic $U 3 D$ viewer}

The generic $U 3 D$ viewer, as shown in Fig. 6, is a more recent development [26]. It allows the visualization of U3D models, which are traditionally embedded in 3D PDF files [21]. So instead of creating huge PDFs, an author can now submit an article together with the corresponding U3D models, and the latter will then be displayed next to the online version of the article on ScienceDirect. Using the U3D viewer, a reader of the article can subsequently browse through all article U3D data sets, zoom into 3D models, rotate and pan 3D models, change the background color, reset initial default view, switch between the "Surface" and "Wireframe" display modes, explore 3D models in stereo with anaglyph glasses, hide/unhide model elements, view the name of each model element one-by-one, and download original data files. This content innovation is broadly applicable across various journals (see, e.g. [19]).

\section{5. $3 D$ Virtual Environments}

The 3D Virtual Environments [13] viewer is the latest development in this joint Elsevier-Kitware project. This viewer allows readers to interactively explore 3D scene reconstructions in real-time. The authors provide their 3D models in the OBJ, PLY or U3D data format, after which the models are then reconstructed next to the online version of the article in ScienceDirect. It is possible to explore Virtual Environments in the full-screen mode and, using the fly-through navigation technique, which allows the 


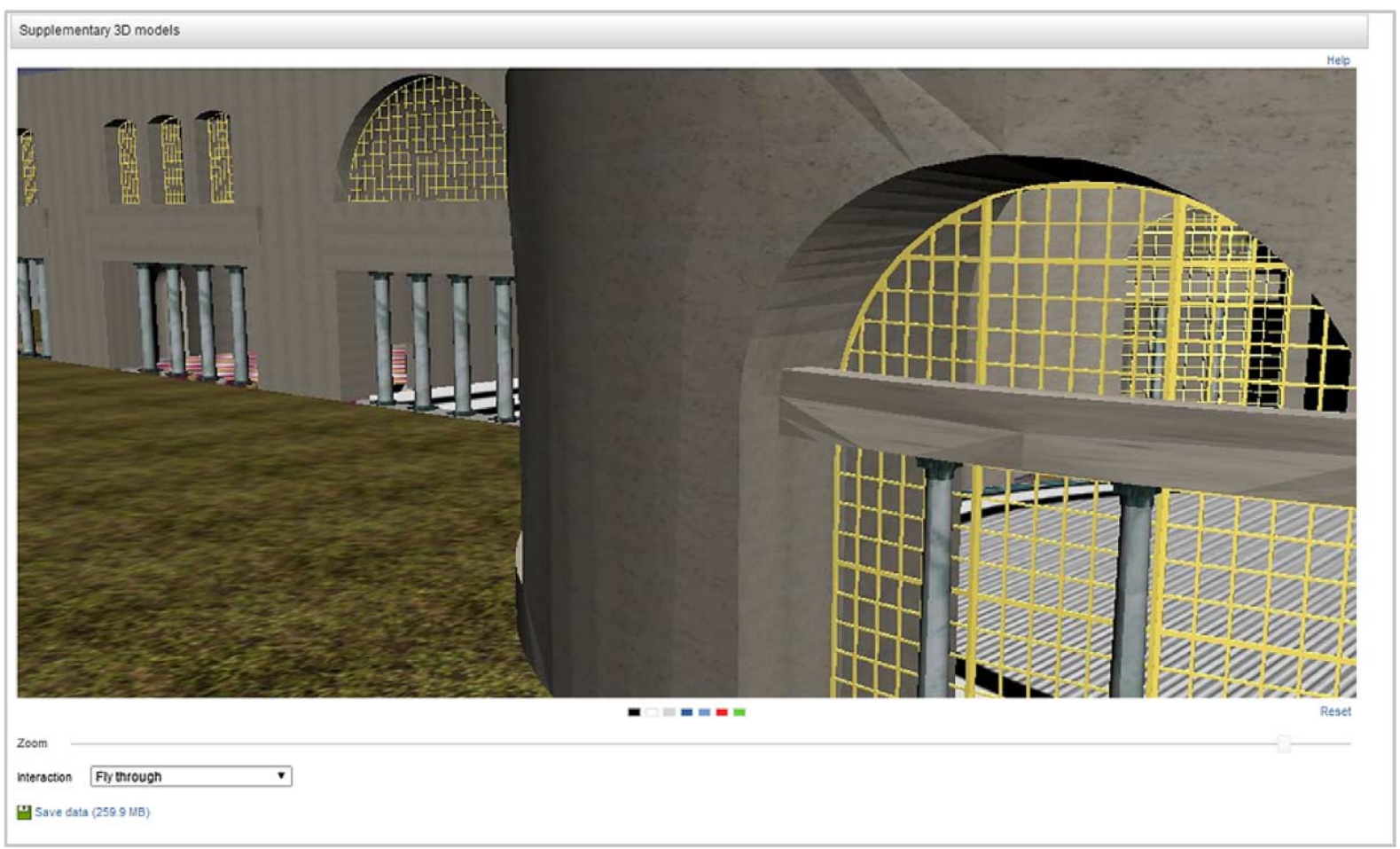

Fig. 7. Example of the 3D Virtual Environment displayed in the full-screen mode. (Colors are visible in the online version of the article; http://dx.doi.org/10.3233/ISU-140721.)

end-user to navigate the Scene View by flying around in first person as it is shown in Fig. 7. In this particular viewer, the scene rendering is performed entirely on a remote server, therefore allowing the user to browse large scenes in real-time. The rendering is performed using a non-photorealistic shading technics called "Eye-Dome Lighting". Furthermore, the combination of various levels of details as well as frustum-culling techniques are used to improve the user's interactivity with the scene.

\section{First author feedback}

To collect user feedback for the 3D visualization functionality that was developed in the 3D project, we conducted a survey amongst the authors who recently published an article with at least one 3D model in one of the Elsevier journals. The invitation to complete the online survey was sent to 100 such authors, from which 34 people completed the survey. Each respondent had to evaluate on ScienceDirect the 3D visualization(s) that was (were) created based on the 3D data that he/she provided with the article. We used SurveyMonkey [25] to conduct the survey and to analyze the data.

Overall, author feedback was positive. Our survey data indicates that $77 \%$ of the respondents are reasonably or very satisfied with the quality of the 3D visualizations created for their 3D data (Fig. 8(a)). The most common reasons for not being satisfied (20\% of the respondents) were: (1) browser compatibility issues and (2) the default size of 3D models. With respect to these two concerns, in the latest release of the Hybrid Visualization Platform all compatibility problems mentioned have been addressed, and we are currently experimenting with several design options to enlarge the default display of 3D models. 

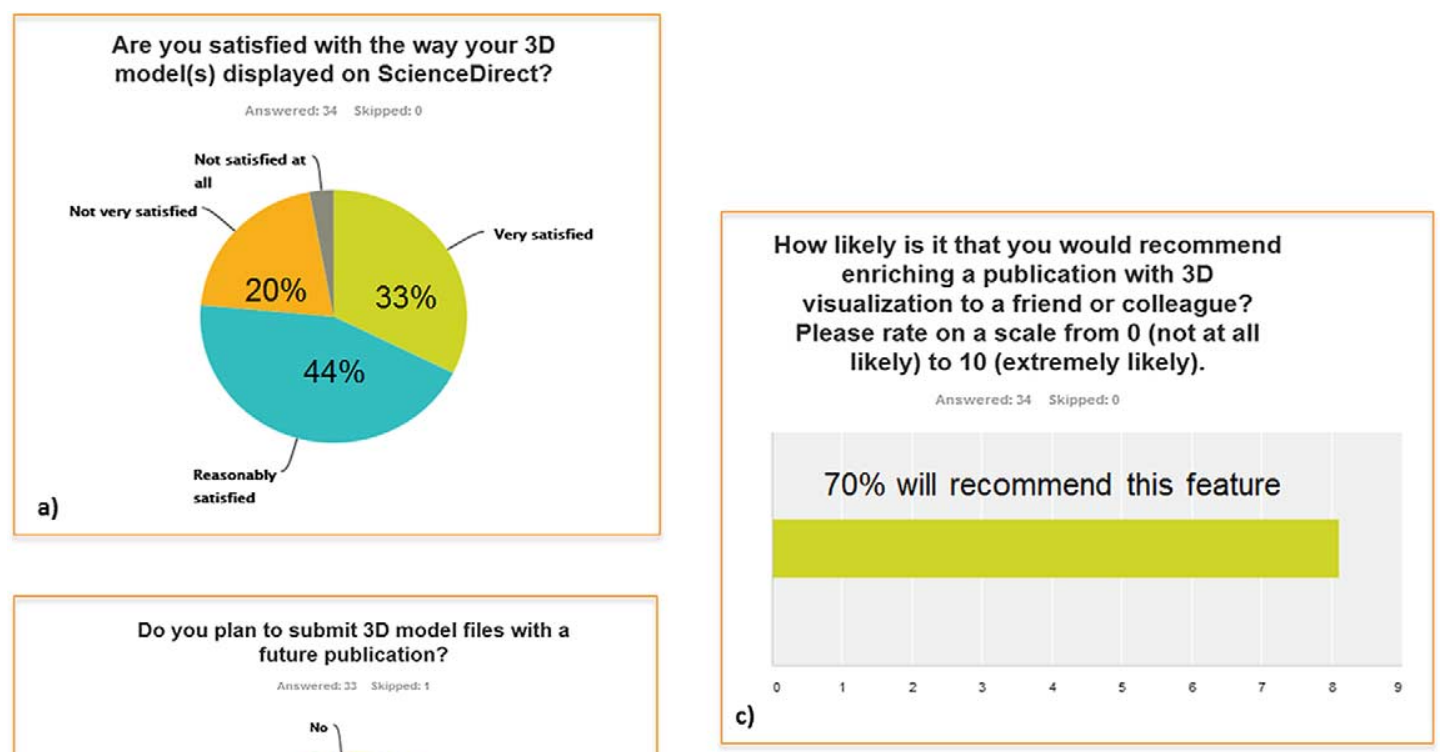

Fig. 8. Survey results with 34 respondents. (Colors are visible in the online version of the article; http://dx.doi.org/ 10.3233/ISU-140721.)

The survey data further established that $76 \%$ of the respondents will again submit 3D models with their next articles (Figure 8(b)). Furthermore, 70\% of the respondents will recommend this new feature to their friends and colleagues (Figure 8(c)).

Here is a small sample of comments from our survey authors about the 3D viewers:

- "This is a really good innovation by the way, well done!"

- "I am convinced that this is the right way to go and I hope this is the first step in providing helpful tools to authors and readers in the future".

- "I think that the publication with $3 D$ visualization is a must in future morphological studies!"

- "Easy to view, it showcases all the possible modes of visualizations which is helpful for any reader".

- "In the moment, we're preparing a manuscript including several interactive 3D models. It will certainly go to the same journal. ... Thanks for this opportunity!"

\section{Conclusion}

The Elsevier's Hybrid Visualization Platform and the first five domain-specific viewers as developed in a joint Elsevier-Kitware project contribute to the ongoing content enrichment effort within Elsevier's journals. Offering interactive visualization tools and techniques, allowing for a better and more accurate interpretation, analysis, and understanding of research is one of the pillars of Elsevier's Article of the 
Future project [9], an on-going program to improve the format of the scientific article [5]. The 3D rendering technology developed and applied allows reader to interact in real-time with large datasets and explore them in a very interactive manner, therefore enhancing the overall reader's experience. Interactive 3D visualization is just a beginning. We plan to expand the existing infrastructure and offer means for online 4D visualizations [15] in online research literature as well.

In this paper, we reviewed five examples of how today's scientific articles outgrew the traditional paper- and print-based format. Fully-interactive archaeological models and 3D brain reconstructions replace the traditional flat images and tables in such articles, providing readers deeper insights through detailed inspection capabilities of the research outcomes. Our first author feedback study showed that scientists are generally happy with the 3D functionality that Elsevier offers.

These developments in visualisation and interactivity are exciting, and can be seen as front runners of fully-executable papers: articles that offer the possibility to re-run and modify complete computational experiments in the context of the article, yet another article enrichment that Elsevier is experimenting with (see, e.g. [22]). 3D visualization of article data and complete executable papers can significantly improve the reader's understanding of the research presented. Elsevier and Kitware will therefore continue to look at ways to further enhance the Hybrid Visualization Platform to improve the scientific publication process. Such enhancements are currently targeting reproducible science and the ability to re-run authors' experiments from a computational point of view. Moreover, the platform could also be extended to support comparison studies between algorithms as well as performing online measurements from accessible datasets.

\section{References}

[1] 3D archaeological pilot homepage: http://www.elsevier.com/3DArchaeologicalModels.

[2] 3D molecular pilot homepage: http://www.elsevier.com/3DMolecularModels.

[3] 3D neuroimaging pilot homepage: http://www.elsevier.com/3DNeuroimaging.

[4] IJ.J. Aalbersberg, S. Atzeni, H. Koers, B. Specker and E. Zudilova-Seinstra, Bringing digital science deep inside the scientific article: the Elsevier article of the future project, LIBER Quarterly: Research Data and New Forms of Scholarly Communication 23(4) (2014), URN:NBN:NL:UI:10-1-116066.

[5] IJ.J. Aalbersberg, F. Heeman, H. Koers and E. Zudilova-Seinstra, Elsevier's article of the future enhancing the user experience and integrating data through applications, Insights: The UKSG Journal 25(1) (2012), 33-43.

[6] J. Ahrens, B. Geveci and C. Law, Paraview: An end-user tool for large data visualization, in: The Visualization Handbook, Elsevier, 2005.

[7] C. Anderson, The end of theory: The data deluge makes the scientific method obsolete, Wired Magazine: 16.07 (2008), available at: http://www.wired.com/science/discoveries/magazine/16-07/pb_theory.

[8] A. Anyuru, Professional WebGL Programming: Developing 3D Graphics for the Web, Wiley, 2012.

[9] Article of the Future: http://www.articleofthefuture.com.

[10] S.S. Chen and J.R. Williamson, Characterization of the ribosome biogenesis landscape in E. coli using quantitative mass spectrometry, Journal of Molecular Biology 425(4) (2013), 767-779.

[11] J.H. Cole, C.P. Boyle, A. Simmons, S. Cohen-Woods, M. Rivera, P. McGuffin, P.M. Thompson and C.H. Fu, Body mass index, but not FTO genotype or major depressive disorder, influences brain structure, Neuroscience 252 (2013), 109-117.

[12] R.W. Cox, J. Ashburner, H. Breman, K. Fissell, C. Haselgrove, C.J. Holmes, J.L. Lancaster et al., A (sort of) new image data format standard: Nifti-1, Human Brain Mapping 25 (2004).

[13] C. Cruz-Neira, D.J. Sandin, T.A. DeFanti, R.V. Kenyon and J.C. Hart, The CAVE: audio visual experience automatic virtual environment, Communications of the ACM 35(6) (1992), 64-72.

[14] Elsevier website: http://www.elsevier.com/.

[15] A.J. Hanson and A.C. Robert, Interactive visualization methods for four dimensions, in: Proceedings of the 4th Conference on Visualization'93, IEEE Computer Society, 1993.

[16] J. Jomier, S.R. Aylward, C. Marion, J. Lee and M. Styner, A digital archiving system and distributed server-side processing of large datasets, Proc. SPIE 7264 (2009), 726413. 
[17] J. Jomier, S. Jourdain, U. Ayachit and C. Marion, Remote visualization of large datasets with MIDAS and ParaViewWeb, in: Web3D Conference 2011, June 2011.

[18] Kitware website: http://www.kitware.com.

[19] B.J. Klußmann-Fricke, L. Prendini and C.S. Wirkner, Evolutionary morphology of the hemolymph vascular system in scorpions: A character analysis, Arthropod Structure \& Development 41(6) (2012), 545-560.

[20] C. Marion, J. Pouderoux, J. Jomier, S. Jourdain, M. Hanwell and U. Ayachit, A hybrid visualization system for molecular models, in: The 18th International Symposium on Web3D Technology, Web3D'13, ACM, June 2013.

[21] A. Newe and T. Ganslandt, Simplified generation of biomedical 3D surface model data for embedding into 3D Portable Document Format (PDF) files for publication and education, PLOS ONE 8(11) (2013), urn:nbn:de:bvb:29-opus4-39761.

[22] P. Nowakowski, E. Ciepiela, D. Harężlak, J. Kocot, M. Kasztelnik, T. Bartyński, J. Meizner, G. Dyk and M. Malawski, The collage authoring environment, Procedia Computer Science 4 (2011), 608-617.

[23] W.J. Schroeder, L.S. Avila and W. Hoffman, Visualizing with VTK: a tutorial, Computer Graphics and Applications, IEEE 20(5) (2000).

[24] E. Smit, Abelard and Héloise: Why data and publications belong together, D-Lib Magazine 17(1,2) (2011).

[25] SurveyMonkey website: https://www.surveymonkey.com/.

[26] U3D pilot homepage: http://www.elsevier.com/about/content-innovation/u3d-models.

[27] G.W. Weber, Another link between archaeology and anthropology: Virtual anthropology, Digital Applications in Archaeology and Cultural Heritage 1(1) (2013), 3-11.

[28] E.V. Zudilova-Seinstra, T. Adriaansen and R. van Liere, Trends in interactive visualization, state-of-the-art survey, in: Advanced Information and Knowledge Processing, Springer, 2009. 